\title{
Investigação da Cinética de Cura por Calorimetria Diferencial Exploratória (DSC) de Resinas Epóxi Preparadas a partir de Óleo de Soja Epoxidado com Diferentes Anidridos e Aminas Terciárias
}

\author{
Ana P. 0. Costa, Annelise E. Gerbase, Cesar L. Petzhold \\ Departamento de Química, UFRGS
}

\begin{abstract}
Resumo: No presente trabalho, utilizou-se a calorimetria diferencial exploratória (DSC) para investigar a cinética da reação de cura de resinas epóxi produzidas a partir da reação do óleo de soja epoxidado (ESO), com os anidridos dodecenilsuccínico (DDS), maleico (MAL), ftálico (FTA), succínico (SUC) e hexahidroftálico (CH), atuando como agentes de cura, e na presença de aminas terciárias como a trietilamina (TEA), a N,N'-dimetilanilina (ARO) e a 1,4- diazobiciclo[2,2,2] octano (DABCO), atuando como catalisadores. A taxa de aquecimento, a natureza química e a estrutura do anidrido e das aminas influenciaram a reação de cura. Os métodos dinâmicos de Kissinger, Ozawa e de Barrett foram utilizados para calcular a energia de ativação dos sistemas onde se variou o anidrido. Observou-se que os anidridos mais reativos foram o DDS e maleico que apresentaram os menores valores de energias de ativação. No estudo da influência do catalisador, utilizou-se o método de Barrett para determinar as energias de ativação das reações com as diferentes aminas. A amina cicloaliafática $(\mathrm{DABCO})$ foi a mais reativa obtendo-se o menor valor de energia de ativação $\left(\right.$ Ea $\left.=51 \mathrm{~kJ} . \mathrm{mol}^{-1}\right)$ e fator préexponencial $\left(\ln \mathrm{A}_{0}=9 \mathrm{~s}^{-1}\right)$.
\end{abstract}

Palavras-chave: DSC, óleo de soja epoxidado, resina epóxi, estudo cinético.

\section{Investigation of the Kinetics of Cure by Differential Scanning Calorimetry (DSC) of Epoxy Resins Prepared from Epoxidized Soybean Oil with Different Anhydrides and Tertiary Amines}

\begin{abstract}
In the present work, differential scanning calorimetry (DSC) was used to investigate the curing reaction kinetics of epoxy resins produced from reaction of epoxidized soybean oil (ESO) with cyclic anhydrides dodecenylsuccinic (DDS), maleic (MAL), phthalic (PA), succinic (SUC) and hexahydrophthalic $(\mathrm{CH})$, in the presence of tertiary amines such as triethylamine (TEA), N,N-dimethylaniline (ARO) and 1,4-diazabicyclo[2,2,2]octane (DABCO). The heating rate, the nature and structure of anhydrides and amines were found to affect the curing reaction. The dynamic methods of Kissinger, Ozawa and Barrett were used to calculate the activation energy of the reactions where different anhydrides were used. DDS and maleic anhydrides were more reactive than the others. In studying the influence from the catalyst, we used the Barret's method to determine the activation energy of the reactions. The 1,4-diazabicyclo[2,2,2]octane amine $(\mathrm{DABCO})$ was the most reactive, showing the lowest activation energy $\left(\mathrm{Ea}=51 \mathrm{~kJ} \cdot \mathrm{mol}^{-1}\right)$ and pre-exponential factor $\left(\ln \mathrm{A}_{0}=9 \mathrm{~s}^{-1}\right)$.
\end{abstract}

Keywords: DSC, epoxidized soybean oil, epoxy resin, kinetic study.

\section{Introdução}

As resinas epóxi fazem parte de uma das mais importantes classes de polímeros termofixos. As inúmeras formas de processamento tornam possível a sua aplicação em diversos segmentos industriais tais como: tintas, adesivos, revestimentos e como matrizes de compósitos devido a suas propriedades químicas e físicas ${ }^{[1-4]}$. Estas resinas têm boas propriedades térmicas, elétricas e mecânicas, embora sejam frágeis e apresentem baixa resistência à propagação de trincas e fraturas em consequência de sua alta densidade de ligações cruzadas. Algumas propriedades mecânicas podem ser significativamente melhoradas com a utilização de óleos vegetais como óleo de soja ${ }^{[5-8]}$ ou óleo de linhaça ${ }^{[9]}$ em sua formulação.

As propriedades físicas e mecânicas das resinas curadas são conhecidas por serem dependentes da estrutura do agente de reticulação, do grau de cura, do tempo e da temperatura de cura $^{[10,11]}$. Através do estudo cinético da reação de polimerização é possível obter informação das condições de processamento e também explicar diferenças entre as propriedades físicas e mecânicas de diferentes sistemas.

A calorimetria diferencial exploratória (DSC) tem sido usada com sucesso no estudo da reação de cura de resinas epóxi visando estimar parâmetros cinéticos ${ }^{[12-14]}$. Os métodos dinâmicos tais como: o método de Borchardt e Daniels ${ }^{[15]}$, de Kissinger ${ }^{[16]}$, de Ozawa $^{[17]}$ e o método Barrett ${ }^{[18]}$, oferecem resultados mais rápidos quando comparados aos métodos isotérmicos.

A maioria dos estudos onde se utiliza a técnica de DSC no monitoramento das reações de cura de resinas epóxi comerciais refere-se a compostos bi, tri e multifuncionais ${ }^{[4,19-21]}$.

Alguns trabalhos relatam a incorporação de óleos vegetais modificados em formulações comerciais com DGEBA ${ }^{[5,22-24]}$. No entanto, existem poucos trabalhos utilizando a técnica de DSC para avaliar a cura de resinas epóxi em que se utiliza um poliepóxido obtido a partir da modificação química de óleos vegetais ${ }^{[25]}$.

A utilização de óleos vegetais epoxidados como matériaprima pela indústria, tem um papel fundamental na manutenção das reservas petrolíferas, na diminuição da dependência dos derivados petroquímicos, além do fato, de que os grupos epóxi presentes em sua estrutura podem estar localizados em posições internas das cadeias carbônicas, possibilitando assim a ocorrência de movimentos moleculares entre as cadeias e com isso o polímero resultante teria maior flexibilidade.

O presente trabalho teve como objetivo principal investigar a cinética de cura de óleo de soja epoxidado (ESO) com diferentes anidridos cíclicos, e na presença de aminas terciárias. Para a

Autor para correspondência: Ana P. O. Costa, Instituto de Química, Universidade Federal do Rio Grande do Sul, Caixa Postal 15003, CEP: 91501-970, 
determinação dos parâmetros cinéticos utilizou-se a técnica de calorimetria diferencial exploratória e foram aplicados os métodos matemáticos dinâmicos de Kissinger ${ }^{[16]}$, Ozawa ${ }^{[17]}$ e Barrett ${ }^{[18]}$ no estudo dos diferentes anidridos. Para o estudo da influência do tipo de catalisador foi aplicado somente o método de Barret.

\section{Parte Experimental}

Os anidridos ftálico (FTA), hexahidroftálico (CH), maleico (MAL) e a amina N,N'-dimetilanilina (ARO) (Aldrich Chemical Co, Milwaukee, USA) foram purificados por métodos padrões antes de serem utilizados. O anidrido succínico (SUC) (Sigma Co, St. Louis, USA) foi recristalizado em clorofórmio. A trietilamina (TEA) (Merck, Darmstadt, Germany) foi destilada antes de ser utilizada. O anidrido dodecenilsuccinico (DDS) (Sigma Co, St. Louis, USA) e a amina 1,4-diazobiciclo[2.2.2] octano (DABCO) (Aldrich Chemical Co, Milwaukee, USA) foram utilizados como recebido.

Utilizou-se óleo de soja epoxidado comercial marca Henkel EDENOL D81 (ESO-D81) (CBM, Cachoeirinha, Rio Grande do $\mathrm{Sul}$ ) com 6-7\% de conteúdo de grupo epóxi, massa molecular média de 935 g.mol ${ }^{-1}$ e aproximadamente 3,8 grupos epóxi por triglicerídeo, correspondendo a 4,1 mmol.g ${ }^{-1}$ de grupos epóxido.

Para o estudo da influência do tipo anidrido na cinética de cura, foram preparadas misturas de ESO-D81 e anidrido, usando a condição estequiométrica entre grupos epóxi e grupos anidrido. $\mathrm{O}$ ESO-D81 e o anidrido foram aquecidos até a temperatura de fusão do anidrido. Após o resfriamento até a temperatura ambiente, a TEA amina foi adicionada na quantidade de 3,3\% mol em relação ao conteúdo epóxido.

Para o estudo da influência do tipo de catalisador na cinética de cura foram utilizadas as mesmas condições reacionais, porém as misturas foram preparadas pela adição do ESO-D81 com o anidrido DDS variando-se a amina terciária.

Para as experiências dinâmicas, pesou-se cerca de $5 \mathrm{mg}$ das misturas não-curadas em porta-amostras de alumínio fechado, que foram submetidos às razões de aquecimento de $1,2,5,10$ e $20^{\circ} \mathrm{C} / \mathrm{min}$, na faixa de temperatura de 30 a $300{ }^{\circ} \mathrm{C}$, sob atmosfera de $\mathrm{N}_{2}$. Em todas as análises fez-se uma segunda corrida a fim de detectar algum material não-reagido. Os ensaios de DSC foram realizados em um equipamento Perkin-Elmer modelo (DSC-4) com sistema de refrigeração até $-40^{\circ} \mathrm{C}$.

Os parâmetros da curva de DSC: $\mathrm{T}_{\text {min }}, \mathrm{T}_{\text {onset }}, \Delta \mathrm{H}$ e $\alpha$ (conversão) foram determinados através do programa do equipamento. Inicialmente foi traçada uma linha de base (LB), a partir da qual se determinou a temperatura inicial $\left(\mathrm{T}_{\mathrm{i}}\right)$ e a temperatura final $\left(\mathrm{T}_{\mathrm{f}}\right)$ do processo de cura exotérmico. A integração da área do pico exotérmico representa o calor total $\left(\Delta \mathrm{H}_{\mathrm{T}}\right)$ envolvido na reação podendo ser expresso em J.g ${ }^{-1}$, $\mathrm{kJ}$ por equivalente de epóxido

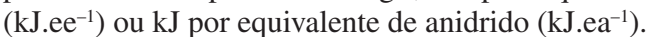

Os parâmetros cinéticos foram determinados usando o método de Barrett $^{[18]}$. Somente foram consideradas para o cálculo dos parâmetros cinéticos as conversões entre 10 e $90 \%$.

Quando se faz estudo cinético de uma reação através de DSC, é necessário supor que a energia envolvida no processo está relacionada com a extensão do consumo de grupos funcionais $(\alpha)$, definido pela Equação 1 que demonstra que o calor parcial liberado na reação é proporcional à extensão da extensão da reação.

$$
\alpha=\frac{\Delta H_{t}}{\Delta H_{t}}=\frac{a}{A}
$$

O grau de cura das resinas correspondente a conversão parcial, foi avaliado utilizando a curva obtida por DSC e calculado como a razão entre o calor parcial da reação em um tempo $t\left(\Delta \mathrm{H}_{\mathrm{t}}\right)$ e o calor total da reação completa $\left(\Delta \mathrm{H}_{\mathrm{T}}\right)$.
Como mostrado na Figura 1, esses parâmetros apresentam uma relação direta com a razão da área parcial (a) a uma dada temperatura (T) e a área total (A) dos picos da curva de DSC, respectivamente.

A constante de velocidade $(\mathrm{k})$ da reação de cura foi estimada a partir da variação de $\alpha$ contra t, utilizando-se relação de Barrett, como mostrado na Equação 2.

$$
k=\frac{d H / d T}{H_{T}-H}
$$

onde $\mathrm{dH} / \mathrm{dt}$ é a variação do calor envolvido na reação ( $\mathrm{J} / \mathrm{min})$.

O método de Barrett pressupõe que a ordem de reação é unitária, no caso a reação entre a resina epóxi e o agente de cura, e assume que a taxa da reação é dependente da temperatura conforme a equação de Arrhenius. A seguinte equação da reta é obtida:

$$
\ln k=\ln A_{0}-\frac{E_{a}}{R T}
$$

onde $\mathrm{k}$ é a constante de velocidade da reação, $\mathrm{A}_{\mathrm{o}} \mathrm{o}$ fator préexponencial, $\mathrm{E}_{\mathrm{a}}$ a energia de ativação, $\mathrm{R}$ a constante universal dos gases e $\mathrm{T}$ a temperatura absoluta.

Através do gráfico ln $\mathrm{k} v$ s. 1/T foi possível determinar a energia de ativação total (Ea) e $\ln \mathrm{A}_{0}$ (fator pré-exponencial) usando o método de Barrett, o qual foi aplicado para a área que compreende a reação exotérmica.

A equação proposta por Kissinger ${ }^{[16]}$ para o cálculo dos parâmetros de Arrhenius é:

$$
\ln \left(\frac{\phi}{\left(T_{p}\right)^{2}}\right)=\ln \left(\frac{A_{0} R}{T}\right)-\frac{E a}{R T_{p}}
$$

onde $\phi$ é a razão de aquecimento; Tp é a temperatura absoluta do pico em cada taxa de aquecimento, $\mathrm{A}_{\mathrm{o}}$ é o fator pré-exponencial de Arrhenius, $\mathrm{R}$ é a constante universal dos gases $\left(8,3145 \mathrm{~J} \mathrm{~mol}^{-1} \mathrm{~K}^{-1}\right)$ e Ea a energia de ativação da reação.

No método de Ozawa ${ }^{[17]}$ os parâmetros cinéticos são obtidos a partir da Equação 5

$$
\ln \phi=\frac{E a}{R T}-\ln A_{0}
$$

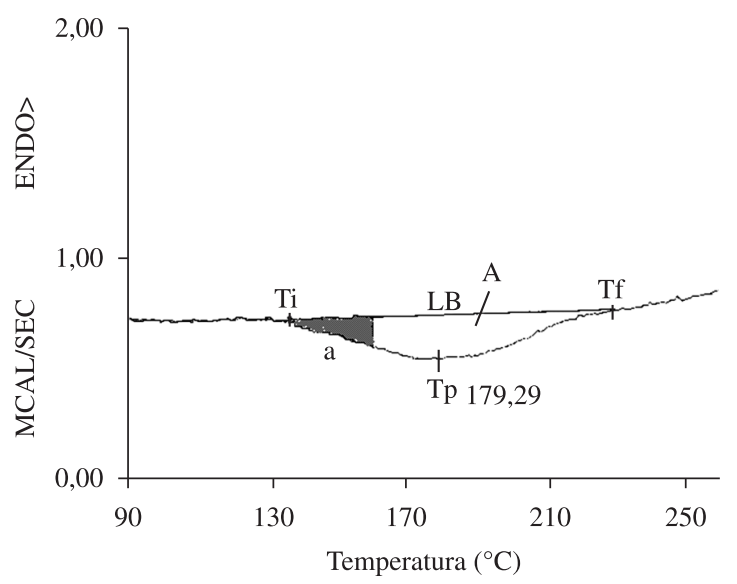

Figura 1. Curva exotérmica típica obtida por DSC para o sistema ESO-D81/ SUC/TEA. ESO-D81 (óleo de soja epoxidado), SUC (anidrido succínico), TEA (trietilamina) na condição estequiométrica e na taxa de aquecimento de $5{ }^{\circ} \mathrm{C} / \mathrm{min} . \mathrm{T}_{\mathrm{i}}=$ temperatura inicial; $\mathrm{T}_{\mathrm{p}}=$ temperatura do pico exotérmico, $\mathrm{T}_{\mathrm{f}}=$ temperatura final, $\mathrm{LB}=$ linha de base, $\mathrm{A}=$ área total, $\mathrm{a}=$ área parcial em uma determinada temperatura. 
onde $\phi$ é a razão de aquecimento, Ea é a energia de ativação da reação, R é a constante universal dos gases $\left(8,3145 \mathrm{~J} \mathrm{~mol}^{-1} \mathrm{~K}^{-1}\right)$ e T a temperatura absoluta.

Nesse método, o logaritmo da taxa de aquecimento $(\phi)$ é plotado em função do inverso da temperatura do pico de transição.

\section{Resultados e Discussão}

No estudo realizado observou-se que os valores de entalpia

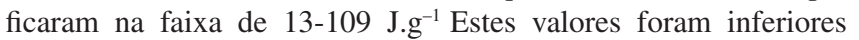
aos das resinas epóxi comerciais DGEBA $^{[26,27]}$, que variam de 322-578 J.g ${ }^{-1}$ e também para sistemas contendo óleo de linhaça ${ }^{[9]}$ que variam de 180-318 J.g-1 . No entanto, este comportamento era esperado, uma vez que, estes materiais têm menor conteúdo de grupos epóxidos para sistemas com massas moleculares próximas.

Gonis et al. ${ }^{[28]}$ avaliaram a influência do comprimento da cadeia carbônica na reação de cura de resinas epóxi do tipo poli(óxido de etileno)diglicidiéter. Os resultados obtidos indicaram que a estrutura da matriz epóxi afeta a quantidade de energia liberada. Deste modo, pode-se supor que os menores valores de entalpia obtidos para os sistemas a base de óleos vegetais sejam devido a fatores estéreos provenientes da estrutura molecular da matriz do óleo epoxidado.

Na Tabela 1 são mostrados os valores de $\Delta \mathrm{H}_{\mathrm{T}}$ e $\mathrm{T}_{\mathrm{p}}$ em diferentes taxas de aquecimento para os diferentes sistemas epóxi estudados. Conforme esperado com o aumento da taxa de aquecimento, a temperatura do pico $\mathrm{T}_{\mathrm{p}}$ aumenta.
A análise de variância foi realizada com esses dados obtendo-se um nível de significância de $95 \%$, o que confirmou que as diferenças encontradas nos valores são significativas $(\mathrm{F}=9,41, \mathrm{p}<0,0005)$.

$\mathrm{Na}$ Tabela 2 são mostrados os resultados referentes ao estudo da influência do tipo anidrido na cinética de cura. A E foi calculada de acordo com o método de Barrett, nos experimentos realizados a $5{ }^{\circ} \mathrm{C} /$ min e pelos métodos de múltiplas varreduras de Osawa e Kissinger. Comparando os resultados obtidos pelos três métodos observou-se que os valores determinados são da mesma ordem de grandeza dos sistemas à base de óleos vegetais epoxidados descritos na literatura ${ }^{[9,29]}$ e que as diferenças observadas nos valores podem ser atribuídas às limitações de cada método. De forma geral, observou-se que a energia de ativação diminui na sequência $\mathrm{CH}>\mathrm{SUC}>\mathrm{PA}>\mathrm{MAL}>\mathrm{DDS}$.

O mecanismo de polimerização epóxi-anidrido na presença de uma de amina terciária como catalisador é bastante complexo, mas a maioria dos autores concorda que o mecanismo de reação é aniônico (Figura 2) ${ }^{[30]}$. Pode-se dizer que a reação é iniciada pela reação entre a amina terciária com o grupo epóxi formando um "zwitterion" que apresenta em sua estrutura um átomo de carbono quaternário e um anion alcóxido (reação 1) e que o processo de propagação ocorre através de uma acilação seguida de uma esterificação (reação 2 e 3 ). Assim, tanto o epóxido como anidrido estão presentes em todas as etapas da reação, em parte como constituintes do alcóxido e em parte como carboxilato, o primeiro pode reagir apenas com anidrido e o último apenas com o epóxido. Assim, uma ordem fracionada poderia surgir em relação ao epóxido que reagiu e anidrido

Tabela 1. Parâmetros cinéticos calculados para o sistema ESO-D81/anidrido/TEA na condição estequiométrica usando diferentes taxas de aquecimento.

\begin{tabular}{|c|c|c|c|c|c|}
\hline Exp. & Sistema & $\begin{array}{c}\phi^{\mathrm{a})} \\
\left({ }^{\circ} \mathrm{C} / \mathrm{min}\right)\end{array}$ & $\begin{array}{c}\mathbf{T}_{\mathrm{p}} \\
\left({ }^{\circ} \mathbf{C}\right)\end{array}$ & $\begin{array}{l}-\Delta \mathbf{H}_{\mathrm{T}} \\
\left(\mathrm{J} . \mathrm{g}^{-1}\right)\end{array}$ & $\begin{array}{c}\ln \mathbf{A}_{0} \\
\left(\mathrm{~s}^{-1}\right)\end{array}$ \\
\hline 1 & ESO-D81/SUC/TEA & 1 & 149 & 196 & 24 \\
\hline 2 & ESO-D81/CH/TEA & 1 & 151 & 188 & 13 \\
\hline 3 & ESO-D81/PA/TEA & 1 & 142 & 226 & 12 \\
\hline 4 & ESO-D81/MAL/TEA & 1 & 116 & 138 & 8 \\
\hline 5 & ESO-D81/DDS/TEA & 1 & 136 & 151 & 10 \\
\hline 6 & ESO-D81/SUC/TEA & 2 & 159 & 164 & 13 \\
\hline 7 & ESO-D81/CH/TEA & 2 & 146 & 46 & 15 \\
\hline 8 & ESO-D81/PA/TEA & 2 & 143 & 76 & 14 \\
\hline 9 & ESO-D81/MAL/TEA & 2 & 122 & 147 & 11 \\
\hline 10 & ESO-D81/DDS/TEA & 2 & 160 & 118 & 12 \\
\hline 11 & ESO-D81/SUC/TEA & 5 & $179 \pm 6$ & $118 \pm 6$ & $21 \pm 4$ \\
\hline 12 & ESO-D81/CH/TEA & 5 & $151 \pm 3$ & $80 \pm 3$ & $26 \pm 5$ \\
\hline 13 & ESO-D81/PA/TEA & 5 & $147 \pm 8$ & $67 \pm 2$ & $17 \pm 1$ \\
\hline 14 & ESO-D81/MAL/TEA & 5 & 1412 & $84 \pm 5$ & $17 \pm 1$ \\
\hline 15 & ESO-D81/DDS/TEA & 5 & $147 \pm 3$ & $46 \pm 4$ & $14 \pm 2$ \\
\hline 16 & ESO-D81/SUC/TEA & 10 & $181 \pm 3$ & $109 \pm 6$ & $28 \pm 1$ \\
\hline 17 & ESO-D81/CH/TEA & 10 & $189 \pm 17$ & $72 \pm 1$ & $14 \pm 1$ \\
\hline 18 & ESO-D81/PA/TEA & 10 & $164 \pm 19$ & $76 \pm 8$ & $10 \pm 2$ \\
\hline 19 & ESO-D81/MAL/TEA & 10 & 1606 & $13 \pm 1$ & $18 \pm 6$ \\
\hline 20 & ESO-D81/DDS/TEA & 10 & $195 \pm 19$ & $42 \pm 1$ & $10 \pm 1$ \\
\hline 21 & ESO-D81/SUC/TEA & 20 & $201 \pm 3$ & $88 \pm 6$ & $30 \pm 1$ \\
\hline 22 & ESO-D81/CH/TEA & 20 & $171 \pm 1$ & $34 \pm 1$ & $14 \pm 2$ \\
\hline 23 & ESO-D81/PA/TEA & 20 & $180 \pm 2$ & $13 \pm 1$ & $26 \pm 6$ \\
\hline 24 & ESO-D81/MAL/TEA & 20 & 1786 & $63 \pm 1$ & $13 \pm 1$ \\
\hline 25 & ESO-D81/DDS/TEA & 20 & $203 \pm 2$ & $26 \pm 1$ & $23 \pm 7$ \\
\hline
\end{tabular}

${ }^{a} \phi=$ taxa de aquecimento; $\mathrm{T}_{\mathrm{p}}=$ temperatura do pico exotérmico; $-\Delta \mathrm{H}_{\mathrm{T}}=$ calor total da reação; $\ln \mathrm{A}_{0}$ = fator pré-exponencial; ESO-D81, óleo de soja epoxidado; SUC, anidrido succínico; $\mathrm{CH}$, anidrido hexahidroftálico; PA, anidrido ftálico; MAL, anidrido maleico; DDS, anidrido dodecenilsuccínico; TEA, trietilamina. 
Tabela 2. Energias de ativação dos sistemas ESO-D81/anidrido/TEA na condição estequiométrica e taxa de aquecimento de $5{ }^{\circ} \mathrm{C} / \mathrm{min}$, calculados por diferentes métodos.

\begin{tabular}{|c|c|c|c|c|c|c|c|}
\hline Exp. & Sistema & $\begin{array}{l}\mathbf{E}_{\mathrm{a}(\text { (Barrett) }}{ }^{\mathrm{a})} \\
\left(\mathbf{k J J} \mathrm{mol}^{-1}\right)\end{array}$ & FC & $\begin{array}{c}\mathbf{E}_{\text {a(Kissinger) }}{ }^{\text {b) }} \\
\left(\mathbf{k J J} \mathrm{mol}^{-1}\right)\end{array}$ & FC & $\begin{array}{c}\mathbf{E}_{\text {a(Osawa) }}{ }^{\mathrm{c})} \\
\left(\mathrm{kJJ}^{2} \mathrm{~mol}^{-1}\right)\end{array}$ & FC \\
\hline 1 & ESO-D81/SUC/TEA & 92 & 0,98 & 89 & 0,98 & 97 & 0,98 \\
\hline 2 & ESO-D81/CH/TEA & 109 & 0,94 & 126 & 0,98 & 133 & 0,98 \\
\hline 3 & ESO-D81/PA/TEA & 76 & 0,93 & 80 & 0,95 & 87 & 0,96 \\
\hline 4 & ESO-D81/MAL/TEA & 76 & 0,98 & 60 & 0,99 & 67 & 0,99 \\
\hline 5 & ESO-D81/DDS/TEA & 67 & 0,94 & 64 & 0,98 & 71 & 0,98 \\
\hline
\end{tabular}

${ }^{a} \mathrm{E}_{\mathrm{a}}$, energia de ativação; FC, fator de correlação; ESO-D81, óleo de soja epoxidado; SUC, anidrido succínico; CH, anidrido hexahidroftálico; PA, anidrido ftálico; MAL, anidrido maleico; DDS, anidrido dodecenilsuccínico; TEA, trietilamina.

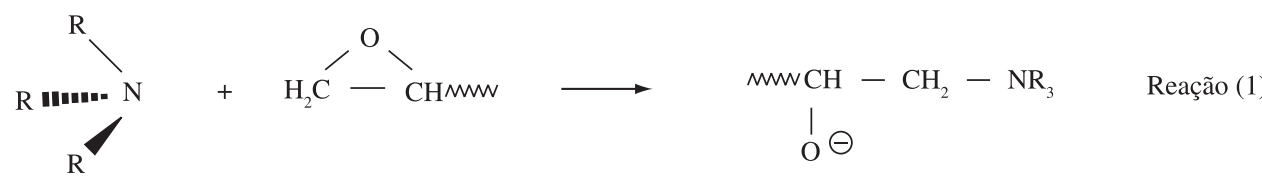<smiles>[R]NC[C@H](C)OC(=O)C=CC(=O)O</smiles>

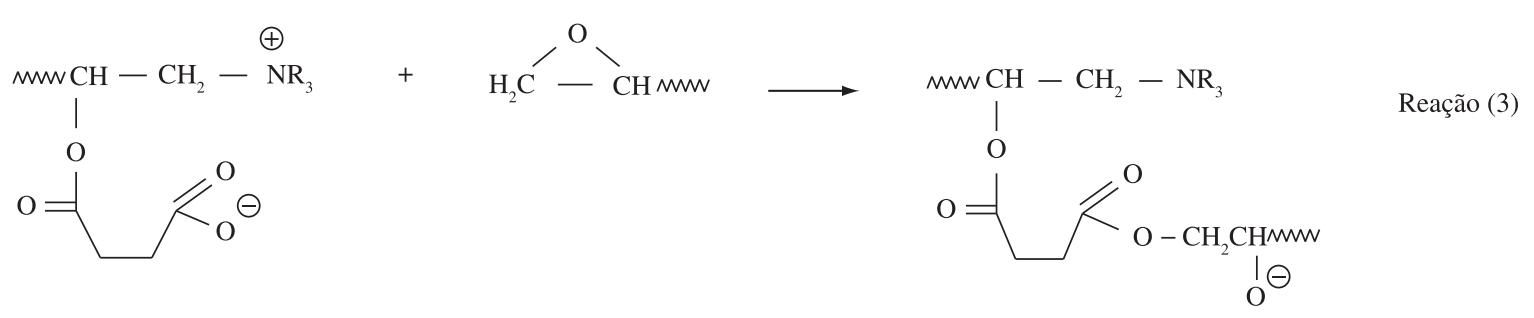

Figura 2. Mecanismo da reação de cura do sistema epóxi/anidrido/amina terciária.

equivalente. Uma ordem fracionada é consistente com o efeito total observado de uma sequência de reações elementares e não uma etapa única reação elementar.

De acordo com o mecanismo descrito acima, a reatividade do anidrido depende de seu caráter eletrofílico e do seu impedimento estéreo $^{[31]}$. Como é mostrado na Figura 2, a etapa de iniciação é independente do tipo de anidrido (reação 1), mas a eletrofilia do anidrido é determinante na segunda etapa (reação 2). Reações de cura mais rápidas são esperadas quando se utiliza grupos anidridos com maior caráter eletrofílico (substituintes retiradores de elétrons). Contudo o impedimento estéreo é o fator mais importante para a reação de reticulação na terceira etapa (reação 3 ) e os anidridos que apresentam segmentos diéster de cadeia flexível reagirão com maior facilidade.

Os valores de $\mathrm{E}_{\mathrm{a}}$ obtidos neste trabalho mostram que a eletrofilia do grupo anidrido é mais importante do que o fator estéreo para a taxa global de cura de reação, pois os anidridos MAL e PA, que são mais eletrofílicos, contendo grupos arila e vinila como substituintes, apresentam maior reatividade (menor $\mathrm{E}_{\mathrm{a}}$ ) do que $\mathrm{SUC}$ e $\mathrm{CH}$, os quais possuem substituintes alquilas ligados ao grupo anidrido. Um comportamento inesperado foi observado para o anidrido DDS, que tem eletrofilia similar ao SUC. A maior reatividade do anidrido DDS pode ser devida ao fato de que esse reagente é líquido à temperatura ambiente, diferente dos demais anidridos que são sólidos, o que facilitaria a mistura com o óleo epoxidado ou também pela presença de impurezas que catalisariam a reação de cura, uma vez que este anidrido foi usado sem purificação prévia.
Tabela 3. Parâmetros cinéticos dos sistemas ESO-D81/DDS/amina, na condição estequiométrica utilizando uma taxa de aquecimento de $5{ }^{\circ} \mathrm{C} / \mathrm{min}$.

\begin{tabular}{|c|c|c|c|c|c|c|}
\hline Catalisador & $\begin{array}{c}\mathbf{T}_{\mathrm{p}} \\
\left({ }^{\circ} \mathbf{C}\right)\end{array}$ & $\begin{array}{c}\Delta \mathbf{H}_{\mathrm{T}} \\
\left(\mathrm{J} \cdot \mathrm{g}^{-1}\right)\end{array}$ & $\begin{array}{c}\mathbf{E}_{\mathrm{a}} \\
\left(\mathrm{kJJ} \cdot \mathrm{mol}^{-1}\right)\end{array}$ & $\begin{array}{r}\ln \mathbf{A}_{0} \\
\left(\mathbf{s}^{-1}\right)\end{array}$ & $\mathbf{C F}$ & pka \\
\hline ARO & 158 & 88 & 80 & 17 & 0,98 & 5,1 \\
\hline TEA & 160 & 118 & 59 & 12 & 0,98 & 10,7 \\
\hline DABCO & 142 & 184 & 51 & 9 & 0,96 & 9,8 \\
\hline
\end{tabular}

ESO-D81, óleo de soja epoxidado; DDS, anidrido dodecenilsuccínico; $\mathrm{T}_{\mathrm{p}}$, temperatura do pico exotérmico; $-\Delta \mathrm{H}_{\mathrm{T}}$, calor total da reação; $\mathrm{E}_{\mathrm{a}}^{\mathrm{p}}$, energia de ativação; $\ln \mathrm{A}_{0}$, fator pré-exponencial; $\mathrm{CF}$, fator de correlação; TEA, trietilamina; ARO,N,N'-dimetilanilina; DABCO,

1,4-diazobiciclo[2.2.2]octano.

A influência do tipo de catalisador foi estudada para os sistemas ESO-D81/ anidrido DDS /amina A Tabela 3 mostra os dados de DSC realizado utilizando uma única taxa de aquecimento de $5{ }^{\circ} \mathrm{C} / \mathrm{min}$ e a energia de ativação calculada utilizando o método de Barrett. Como pode ser observado, o calor de entalpia aumenta na ordem ARO $<$ TEA $<$ DABCO enquanto que $\mathrm{E}_{\mathrm{a}}$ diminui, mostrando que a ordem de reatividade encontrada é $\mathrm{ARO}<\mathrm{TEA}<\mathrm{DABCO}$.

É esperado que quanto maior a basicidade de uma amina maior seja a reatividade do sistema. No entanto, a ordem de reatividade das aminas estudadas não segue a ordem de basicidade, ARO $(\mathrm{pka}=5,1)$, DABCO $(\mathrm{pka}=9,8)$ e TEA $(\mathrm{pka}=10,7)$. O efeito catalítico de uma amina está relacionado não só à sua basicidade, mas também ao impedimento estéreo do par de elétrons não compartilhado do átomo de nitrogênio. Assim se explicaria maior 
reatividade da amina DABCO que apesar de não ter a maior basicidade apresenta menor impedimento estéreo. A amina ARO, por outro lado, apresenta a menor basicidade, ou seja, o par isolado de elétrons que se liga ao próton está menos disponível em virtude da possibilidade de entrar em ressonância com o anel aromático tornando esta amina o catalisador menos efetivo para o sistema estudado.

\section{Conclusões}

Polímeros epoxídicos foram obtidos a partir da reação do óleo de soja epoxidado com os anidridos ftálico (FTA), succínico (SUC), maleico (MAL), hexahidroftálico (CH), dodecenilsuccínico (DDS) utilizando aminas terciárias como catalisadores, trietilamina (TEA), 1,4-diazobiciclo[2,2,2] octano (DABCO), N,N'-dimetilanilina (ARO).

O estudo cinético da reação de cura por DSC mostrou ser influenciado pela natureza do anidrido e da amina. Os valores determinados para os parâmetros cinéticos são da mesma ordem de grandeza dos valores encontrados na literatura para sistemas a base de óleos vegetais.

Em relação ao anidrido observou-se que o caráter eletrofílico do anidrido é responsável pela reatividade e conversão da reação. Os anidridos maleico e ftálico apresentaram maior reatividade que os anidridos hexahidroftálico e succínico.

Em relação às aminas terciárias observou-se a influência da basicidade e do impedimento estéreo, sendo a DABCO a mais reativa e a ARO a menos reativa.

\section{Agradecimentos}

Os autores agradecem a CAPES (Coordenadoria de Aperfeiçoamento de Pessoal de Ensino Superior) pelo suporte financeiro e ao Prof. Dr. Ricardo Baumhardt Neto pela ajuda na análise estatística.

\section{Referências Bibliográficas}

1. Plazek, D. J. \& Choy, I. - J. Polym. Sci: Part B: Polym. Phys., 24, p.1303 (1986).

2. Shim, J. S.; Lee, W. \& Jang, J. - Polym. Bull., 25, p.669 (1991).

3. Banks, L. \& Ellis, B. - Polymer, 23, p.1466 (1982).

4. Xiao, F.; Sun, Y.; Xiu, Y. H.; Wong, C. P. - J. Appl. Polym. Sci., 104, p.2113 (2007).

5. Park, S.-J.; Jin, F.-L. \& Lee, J.-R. - Mater. Sci. Eng., A374, p.109 (2004).

6. Zengshe, L.; Erhan, S. Z.; Akoh, C. C. \& Barton, F. E. - J. Agric. Food Chem., 54, p.2134 (2006).
7. Güner, F. S.; Yagci, Y. \& Erciyes, A. T. - Prog. Polym. Sci., 31, p.633 (2006).

8. Zhou, J.; Chandrashekhara, K.; Flaningan, V. \& Kapila, S. - J. Appl. Polym. Sci., 91, p.3513 (2004).

9. Boquillon, N. \& Fringant, C. - Polymer, 41, p.8603 (2000).

10. Gerbase, A. E.; Petzhold, C. L. \& Costa, A. P. O. - J. Am. Oil. Chem. Soc., 79, p.797 (2002).

11. Rosu, D.; Mustata, F. \& Cascaval, C. N. - Thermochim. Acta, 370, p.105 (2001).

12. Kim, S. \& Lu, M. - J. Appl. Polym. Sci., 71, p.2401 (1999).

13. Boey, F. Y. C. \& Qiang, W. - Polymer, 41, p.2081 (2000).

14. Andrade, H. P. C.; Diniz, M. F.; Azevedo, M. F. P.; Cassu, S. N.; Lourenço, V. L. \& Dutra, R. C. L. - Polímeros,18, p.359 (2008).

15. Borchardt, H. J. \& Daniels, F. J. - Am. Chem. Soc., 79, p.41 (1956).

16. Kissinger, H. E. - Anal. Chem., 29, p.17 (1957).

17. Ozawa, T. - J. Therm. Anal. Calorim., 2, p.301 (1970).

18. Barrett, K. E. J. - J. Appl. Polym. Sci., 11, p.1617 (1967).

19. Lee, S.-H.; Jang, J. \& Oh, J. H. - Polymer, 42, p.8339 (2001).

20. Maity, T.; Samanta, B. C.; Dalai, S. \& Banthia, A. K. - Mater. Sci. Eng. A, 464, p.38 (2007).

21. Mustata, F. \& Bicu, I. - J. Opt. Adv. Mater., 9, p.931 (2007),

22. Parzuchowski, P. G.; Jurczyk-Kowalska, M.; Ryskowska, J. \& Rokicki, G. - J. Appl. Polym. Sci.,102, p.2904 (2006).

23. Czub, P. - Macromol. Symp., 242, p.60, (2006).

24. Jin, F-L. \& Park, S-J. - Polym. Int., 57, p.577 (2008).

25. Shabeer, A.; Garg, A.; Sundararaman, S.; Chandrashekhara, K.; Flanigan, V. \& Kapila, S. - J. Appl. Polym. Sci., 98, p.1772 (2005).

26. Miranda, M. I. G.; Bica, C. I. D.; Samios, D. \& Tomedi, C. - Polymer, 38, p.1017 (1997).

27. Costa, M. L.; Botelho, E. C.; Paiva, J. M. F. \& Rezende, M. C. - Mater. Res., 8, p.317 (2005).

28. Gonis, J.; Simon, G. P. \& Cook, W. D. - J. Appl. Polym. Sci., 72, p.1479 (1999).

29. Patel, R. D.; Patel, R. G. \& Patel, V. S. - Angew. Makrom. Chem., 155, p.57 (1987).

30. Matejka, L.; Lövy, J.; Pokornÿ, S.; Bouchal, K. and Dusek, K. - J. Polym. Sci.: Part A: Polym. Chem., 21, p.2873 (1983).

31. Mark, H. F.; Bikales, N.; Overberger, C. G.; Menges, G. \& Kroschwitz, I. J. "Emulsion polymerization to fibers" in: Manufacture encyclopedia of polymer science and engineering, John - Wiley, New York (1986).

Enviado: $24 / 03 / 10$

Reenviado: $30 / 09 / 10$

Aceito: $02 / 10 / 10$

DOI: $10.1590 / \mathrm{S} 0104-14282011005000022$ 